\title{
Randomised trial of chronic supplementation with a nutraceutical mixture in subjects with non-alcoholic fatty liver disease
}

\author{
Chiara Cerletti $^{1}{ }^{*} \dagger$, Mario Colucci ${ }^{2} \dagger$, Marianna Storto $^{3}$, Fabrizio Semeraro ${ }^{2}$, Concetta T. Ammollo ${ }^{2}$, \\ Francesca Incampo ${ }^{2}$, Simona Costanzo ${ }^{1}$, Giuseppe De Bartolomeo ${ }^{4}$, Piero Portincasa ${ }^{5}$, Michele Barone ${ }^{6}$, \\ Augusto Di Castelnuovo ${ }^{7}$, Nicola Semeraro ${ }^{2}$, Licia Iacoviello ${ }^{1,8}$ and Giovanni de Gaetano ${ }^{1}$ \\ ${ }^{1}$ Department of Epidemiology and Prevention, IRCCS Neuromed, Pozzilli (IS), Italy \\ ${ }^{2}$ Laboratory for Haemostasis and Thrombosis, Department of Biomedical Sciences and Human Oncology, University "Aldo \\ Moro", Bari, Italy \\ ${ }^{3}$ Clinical Pathology Unit, IRCCS Neuromed, Pozzilli (IS), Italy \\ ${ }^{4} U$.O. Internal Medicine, Ospedale "F. Veneziale", Isernia, Italy \\ ${ }^{5}$ Clinica Medica "A. Murri", Department of Biomedical Sciences and Human Oncology, University "Aldo Moro", Bari, Italy \\ ${ }^{6}$ Section of Gastroenterology, Department of Emergency and Organ Transplantation, University "Aldo Moro", Bari, Italy \\ ${ }^{7}$ Mediterranea Cardiocentro, Napoli, Italy \\ ${ }^{8}$ Department of Medicine and Surgery, Research Center in Epidemiology and Preventive Medicine (EPIMED), University of \\ Insubria, Varese, Italy
}

(Submitted 5 April 2019 - Final revision received 19 September 2019 - Accepted 20 September 2019)

\section{Abstract}

A mixture of natural ingredients, namely, DHA, phosphatidylcholine, silymarin, choline, curcumin and D- $\alpha$-tocopherol, was studied in subjects with non-alcoholic fatty liver disease (NAFLD). Primary endpoints were serum levels of hepatic enzymes, and other parameters of liver function, the metabolic syndrome and inflammation were the secondary endpoints. The coagulation-fibrinolysis balance was also thoroughly investigated, as NAFLD is associated with haemostatic alterations, which might contribute to increased cardiovascular risk of this condition. The present study involved a double-blind, randomised, multicentre controlled trial of two parallel groups. Subjects with NAFLD (18-80 years, either sex) received the active or control treatment for 3 months. All assays were performed on a total of 113 subjects before and at the end of supplementation. The hepatic enzymes aspartate aminotransferase (AST), alanine aminotransferase and $\gamma$-glutamyl transpeptidase decreased from 23.2 to $3.7 \%$ after treatment, only the AST levels reaching statistical significance. However, no differences were found between control and active groups. Metabolic and inflammatory variables were unchanged, except for a slight (less than 10\%) increase in cholesterol and glucose levels after the active treatment. Coagulation-fibrinolytic parameters were unaffected by either treatment. In conclusion, chronic supplementation with the mixture of dietary compounds was well tolerated and apparently safe in NAFLD subjects. The trial failed to demonstrate any efficacy on relevant physiopathological markers, but its protocol and results may be useful to design future studies with natural compounds.

Key words: Nutraceuticals: Non-alcoholic fatty liver disease: Randomised controlled trials: Coagulation-fibrinolysis: Vascular risk

Non-alcoholic fatty liver disease (NAFLD) is the most common cause of elevated plasma levels of hepatic enzymes in Western industrialised countries, its worldwide prevalence being 6-35\%. In Europe, the median prevalence is $26 \%$, with wide variations in different populations or in subjects at higher risk, such as obese or type 2 diabetes, reaching peaks of $50-70 \%(1-3)$
The NAFLD natural history is associated with a higher cerebroand cardiovascular risk, not only in diabetics but also in healthy subjects $^{(4)}$; in view of its high prevalence, NAFLD treatment and prevention is of great interest from a public health point of view. At present, the main guidelines recommend lifestyle changes, including exercise and correct diet to promote weight loss, while no conclusive evidence is available of drug treatments capable of

Abbreviations: AST, aspartate aminotransferase; NAFLD, non-alcoholic fatty liver disease; TAFI, thrombin activatable fibrinolysis inhibitor.

* Corresponding author: Chiara Cerletti, fax +39-0865-927575, email chiara.cerletti@neuromed.it

$\dagger$ These authors contributed equally to this work and are co-first authors. 
correcting elevated aminotransferase levels and preventing liver steatosis progression in subjects with NAFLD ${ }^{(5)}$.

The multifactorial pathogenesis of NAFLD suggested the use of different nutraceutical elements, and clinical research is ongoing on that direction ${ }^{(6)}$. The most used substances, alone or in combination, are silymarin from Cardo marianum, vitamin $\mathrm{E}$ and other antioxidants, or $n-3$ PUFA. A recent clinical trial showed an improvement in hepatic function among NAFLD subjects treated with a combination of silymarin, phosphatidylcholine and vitamin $\mathrm{E}^{(7)}$. More studies are, however, needed because until now no health claim has been approved by the European Food Safety Authority (EFSA) for improving hepatic function by nutraceuticals. Choline-containing foods may only be labelled as 'maintenance of normal liver function (ID 1501)'(8).

The aim of the present study was to evaluate the effect of a mixture of different natural ingredients, namely, DHA, phosphatidylcholine, silymarin, choline, curcumin and $\mathrm{D}-\alpha$-tocopherol (vitamin E), on the elevated plasma levels of hepatic enzymes in subjects with NAFLD.

Active ingredients were selected based on the clinical evidence of their hepatoprotective activities ${ }^{(6)}$ and on the in vitro evidence of a synergistic beneficial effect of the nutraceutical mixture on lipid accumulation and liver oxidative stress damage ${ }^{(9)}$.

Other parameters of liver function, the metabolic syndrome and inflammation were evaluated as secondary endpoints of the study. Moreover, considering that NAFLD is associated with alterations of the haemostatic system, which might contribute to the increased cardiovascular and thrombotic risk seen in this condition ${ }^{(10-12)}$, the effect of the nutraceutical mixture on coagulation-fibrinolysis balance was investigated in depth, using global functional assays and specific markers of hypercoagulability.

\section{Methods}

This article conforms to the Consolidated Standards of Reporting Trials consortium ${ }^{(13)}$.

\section{Trial design}

The present study was a double-blind, randomised, multicentre controlled trial of two parallel groups. The trial, coordinated by the IRCCS Neuromed, was performed at three different sites: one at the Ospedale 'Veneziale' of Isernia, Italy, and two at the University Hospital, Azienda Ospedaliero-Universitaria Policlinico of Bari, Italy. It was conducted according to the guidelines laid down in the Declaration of Helsinki, and its protocol was approved by the Ethical Committee of the IRCCS Neuromed, followed by each recruitment site's Ethical Committee, namely, the Azienda Sanitaria Regionale del Molise and the Azienda OspedalieroUniversitaria Policlinico of Bari. All participating subjects signed a written informed consent previously approved by the ethical committees. The study was registered in clinicaltrial.gov with identifier NCT 02369536 (https://clinicaltrials.gov/ct2/show/NCT02369536).

\section{Participants}

Subjects of either sex, 18-80 years old, with NAFLD were recruited in the study. They did not manifest nor had previously manifested any liver-related clinical symptoms but simply had altered laboratory and instrumental tests as compared with normal ranges.

Inclusion criteria were ultrasound alterations typical of mild or moderate hepatic steatosis ${ }^{(14)}$ and serum levels higher than normal (i.e. above the upper limit of each laboratory) for at least one of the three liver enzymes, namely, aspartate aminotransferase (AST), alanine aminotransferase (ALT) and $\gamma$-glutamyl transpeptidase $(\gamma$-GT).

Exclusion criteria were alcohol abuse history, use of drugs associated with hepatic steatosis development, malnutrition, alcoholic liver disease, chronic hepatic disease of different aetiologies (autoimmune, primary biliary cholangitis, primary sclerosing cholangitis, hereditary haemochromatosis, Wilson's disease, $\alpha-1$ antitrypsin deficiency, coeliac disease), severe renal, cardiac or respiratory failure, malignant tumours, intolerance to the components of the active ingredients of the formulation, women who were pregnant or had planned for pregnancy in the following 3 months or were breast-feeding, and subjects who refused to sign informed consent.

According to the current guidelines ${ }^{(15)}$, alcohol abuse was considered a weekly intake $>210 \mathrm{~g}$ by men and $>140 \mathrm{~g}$ by women, corresponding to $>2-3$ and $>1-2$ small glasses of wine/d, respectively.

\section{Interventions}

The subjects eligible for the study were randomised (see below) to receive the active or the control treatment, in two capsules once a day, for 3 months.

During the recruitment visit, all subjects received appropriate recommendations about dietary and physical activity lifestyle, according to each site protocols. Recommendations mainly focused on physical activity and healthy diet ${ }^{(15)}$, in particular through adhesion to Mediterranean $\operatorname{diet}^{(16)}$.

The study product was a mixture of active ingredients, formulated as soft gel capsules, each composed of fish oil containing $70 \%$ DHA (250 mg), phosphatidylcholine concentrated in sunflower oil $(150 \mathrm{mg})$, silymarin $(75 \mathrm{mg})$, choline bitartrate (35 mg), curcumin (35 mg) and D- $\alpha$-tocopherol (10 mg), for a total of $830 \mathrm{mg}$. The total content of choline is $21.5 \mathrm{mg}$ per capsule $(43 \mathrm{mg} / \mathrm{d})$, an amount relatively low as compared with the minimal amount recommended by the health claim of EFSA $(82.5 \mathrm{mg} / \mathrm{d})$, corresponding to $15 \%$ of the median daily intake of an adult subject. The comparator capsules (control) contained the formulation excipients and the same amount of choline present in the active mixture (in the form of bitartrate salt). This 'restrictive' choice was made to exclude the possibility of any positive effect of the mixture that could be attributed to choline, which has an EFSA-recognised health claim for maintenance of normal liver function. Both the active and the control capsules were prepared and provided by Bouty S.p.A.

\section{Clinical evaluation}

At the first visit (T0), clinical and pharmacological anamnesis and anthropometric and clinical measures were collected for each subject. The main risk factors for CVD were recorded: diabetes (blood glucose $>6.94 \mathrm{mmol} / \mathrm{l}$ ), hypertension (systolic blood pressure SBP $>140$ and/or diastolic DBP $>90 \mathrm{mmHg}$ or antihypertensive 
treatment), hypercholesterolaemia (serum cholesterol $\geq 5 \cdot 17$ $\mathrm{mmol} / \mathrm{l}$ or anticholesterol treatment), hypertriacylglycerolaemia (serum TAG $\geq 1.69 \mathrm{mmol} / \mathrm{l}$ ), sedentary and smoking habits (exsmokers for more than 1 year were considered as non-smokers), alcohol consumption. BMI was calculated as $\mathrm{kg} / \mathrm{m}^{2}$. Waist (umbilical) circumference was measured at a level midway between the lower rib margin and iliac crest. NAFLD fibrosis score (NFS) was calculated to evaluate the hepatic fibrosis state of each subject ${ }^{(17)}$. A weekly food diary was administered to participants in order to detect changes in eating habits during the study.

The clinical visit was repeated at the end of treatment (T1). Fasting venous blood for haematological and chemical analyses (see below) was collected at T0 and T1; serum and citrated plasma were prepared and stored at $-20^{\circ} \mathrm{C}$ until assay.

\section{Outcomes}

Primary endpoints were serum levels of the hepatic enzymes AST, ALT and $\gamma$-GT; secondary endpoints were liver function markers (direct and indirect bilirubin), metabolic syndrome parameters (cholesterol, TAG, glucose and insulin), C-reactive protein (CRP) and changes in plasma coagulation-fibrinolysis assays (see below).

All measurements were done at completion of the study and were centralised in two laboratories. Biochemical analyses, including re-evaluation of hepatic enzymes and assay of metabolic variables and CRP were performed in the Neuromed Laboratory using standard photometric methods. Insulin was measured by chemiluminescence test and insulin-resistance (homeostatic model assessment of insulin resistance) was calculated $^{(18)}$. Plasma coagulation and fibrinolysis assays were performed in the Laboratory for Haemostasis and Thrombosis, Department of Biomedical Sciences and Human Oncology, University 'Aldo Moro' of Bari. Thrombin generation, which reflects the overall plasma clotting potential, was studied by the calibrated automated thrombinography method as described $^{(19)}$. Plasma clot lysis, which reflects the overall fibrinolytic capacity, was evaluated by a turbidimetric assay ${ }^{(20)}$. Thrombin activatable fibrinolysis inhibitor (TAFI) was measured by a commercial functional assay (Stachrom TAFI; Diagnostica Stago). The following plasma biomarkers were assayed by commercially available ELISA kits: prothrombin fragment $1+2$ $(\mathrm{F} 1+2$, a marker of in vivo thrombin generation) by Enzygnost F1 + 2 ELISA (Dade Behring); D-dimer, a specific fragment derived from the lysis of cross-linked fibrin, by HemosIL AcuStar D-dimer; Instrumentation Laboratory; tissue plasminogen activator (t-PA) and plasminogen activator inhibitor-1 (PAI-1) by Imubind Plasma t-PA ELISA and Imubind Plasma PAI-1 ELISA, respectively (Sekisui Diagnostics $\mathrm{GmbH}$ ); activated TAFI (TAFIa/ai, a combination of TAFIa and its inactive derivative TAFIai) by a specific ELISA (Asserachrom TAFIa/ai; Diagnostica Stago); plasmin- $\alpha 2-$ antiplasmin complex (PAP, a marker of in vivo plasmin generation) by Technozyme PAP (Technoclone $\mathrm{GmbH}$ ).

All measurements were performed by trained personnel blinded to participant identifying information.

Compliance to treatments was achieved by asking the participants to return all unused medications and empty boxes at the end of the trial. They were also asked about the days of missing treatment.

\section{Sample size}

Sixty subjects per group were estimated to allow to detect differences between the two groups equal to $54 \%$ of sD of the mean of the investigated biomarkers with $80 \%$ potency and $\alpha=0.05$. Concerning the primary endpoints, our sample was sized to detect differences greater than $8.3 \mathrm{IU} / \mathrm{l}$ for AST, 13.5 IU/1 for ALT and $16 \cdot 2 \mathrm{IU} / 1$ for $\gamma$-GT (20, 20 and $22 \%$ of the means, respectively).

\section{Randomisation}

The random allocation sequence to treatment was computer based per blocks of four or six subjects stratified for each recruiting centre; it was generated by the principal investigator (PI)'s statistician and forwarded to each recruitment centre.

The doctor responsible for each recruitment centre enrolled participants and assigned them to interventions, according to the allocation sequence received by the PI of the trial.

\section{Blinding}

Participants, doctors, technicians and statisticians who participated or evaluated the study outcomes were blinded to the supplements. Active and control capsules were identical for organoleptic properties and coded as A and B by the producer. Only after completion of statistical analyses of the trial results, the company who provided the capsules opened the blinding.

\section{Statistical methods}

Data were analysed according to the intention-to-treat approach. Results are presented as mean and SD or median and interquartile range, depending on distribution, as assessed by the D'AgostinoPearson test. Differences between basal values in control and active groups and between T $1 v$. T0 \% changes in the two groups were assessed by ANOVA and by Fisher's exact test. Non-normally distributed data were log transformed before analysis.

Additional analyses for composite endpoints were performed using multivariate ANOVA (MANOVA), and for 'intra-subject' (T1 $v$. T0) changes paired data analysis was used. Statistical analyses were performed using the SAS/STAT software, version 9.4 of the SAS System for Windows@2009.

\section{Results}

\section{Participant flow}

A total of 126 participants from three centres were eligible for the study, which was assessed by the willingness to participate in the trial and to sign the informed consent. They were randomly allocated to the active ( $n$ 62) or the control ( $n$ 64) supplementation groups. The subjects lost at follow-up were seven in the active group and six in the control group, for the following reasons: bone fracture (1), relocation to another city (1), discontinued supplementation due to dermatitis (1), unmotivated personal decision (4) in the active group; discontinued supplementation 
Table 1. General and clinical characteristics of subjects included in the study

(Mean values and standard deviations; numbers of subjects and percentages)

\begin{tabular}{|c|c|c|c|c|c|}
\hline \multirow{3}{*}{$\frac{\text { Variable }}{\text { Age (years), mean and SD }}$} & \multicolumn{4}{|c|}{ Treatment } & \multirow{3}{*}{$\frac{P}{0.30}$} \\
\hline & \multicolumn{2}{|c|}{ Active $(n 55)$} & \multicolumn{2}{|c|}{$\begin{array}{c}\text { Control } \\
(n 58)\end{array}$} & \\
\hline & $55 \cdot 7$ & $12 \cdot 2$ & 53.4 & $11 \cdot 3$ & \\
\hline Women, $n$ and \% & 20 & 36 & 19 & 33 & 0.70 \\
\hline CVD, $n$ and \% & 15 & 27 & 9 & 16 & $0 \cdot 17$ \\
\hline Tumours, $n$ and $\%$ & 2 & 4 & 4 & 7 & 0.60 \\
\hline Renal disease, $n$ and \% & 5 & 9 & 5 & 9 & 1.00 \\
\hline Pulmonary disease, $n$ and $\%$ & 4 & 7 & 2 & 3 & 0.43 \\
\hline Diabetes, $n$ and \% & 16 & 29 & 11 & 19 & 0.27 \\
\hline Hypertension, $n$ and $\%$ & 25 & 45 & 21 & 36 & 0.34 \\
\hline Hypercholesterolaemia, $n$ and $\%$ & 24 & 44 & 20 & 34 & 0.32 \\
\hline Hypertriacylglycerolaemia, $n$ and \% & 18 & 33 & 16 & 28 & 0.68 \\
\hline \multicolumn{6}{|l|}{ Medications, $n$ and \% } \\
\hline Antihypertensive & 22 & 40 & 21 & 36 & 0.85 \\
\hline Statins & 11 & 20 & 5 & 9 & 0.11 \\
\hline Antidiabetic & 12 & 22 & 7 & 12 & 0.21 \\
\hline Aspirin & 7 & 13 & 14 & 24 & 0.15 \\
\hline Smokers, $n$ and $\%$ & 14 & 25 & 17 & 29 & 0.68 \\
\hline Sedentary habit, $n$ and $\%$ & 25 & 45 & 25 & 43 & 0.85 \\
\hline Alcohol consumption*, $n$ and \% & 9 & 16 & 5 & 9 & 0.26 \\
\hline NAFLD fibrosis score, mean and SD & $-1 \cdot 29$ & 1.69 & -1.23 & 1.68 & 0.86 \\
\hline
\end{tabular}

NAFLD, non-alcoholic fatty liver disease.

* Less than $210 \mathrm{~g} /$ week for men and $140 \mathrm{~g} /$ week for women.

for faecal colour abnormality (green faeces, 1) and unmotivated personal decision (5) in the control group. The final number of subjects analysed was fifty-five and fifty-eight, in the active and control groups, respectively.

\section{Recruitment}

Subject recruitment started on 18 August 2015, and ended on 19 July 2016. Follow-up visits occurred from 19 November 2015 to 13 October 2016. The recruitment had to be completed by July 2016 in order to meet the project deadline.

\section{Baseline data}

General and clinical characteristics were similar in the active ( $n 55)$ and control ( $n$ 58) supplementation groups (Table 1). Overall, a relatively high proportion of study participants were carriers of risk factors for CVD, such as diabetes, hypertension, or hypercholesterolaemia, hypertriacylglycerolaemia, or had unhealthy lifestyles, smokers being more than $25 \%$ and people with sedentary habits being about $45 \%$; average BMI was $30 \mathrm{~kg} / \mathrm{m}^{2}$, with about $50 \%$ of subjects obese (Table 2). Similarly, no difference was found between the two groups with regard to anthropometric variables, medications (Table 2), blood pressure and complete blood cell counts (data not shown). The NFS was on average low and similar for the two treatment groups. Only a minority (7\%) of participants yielded an NFS compatible with severe fibrosis or cirrhosis. None of the subjects had signs of advanced liver disease.

\section{Outcomes}

Table 2 reports the results on primary and secondary endpoints of the trial. No differences were observed in the baseline levels of the hepatic enzymes, AST, ALT and $\gamma$-GT between the two treatment groups, confirming a successful randomisation. After treatment, both groups displayed a decrease in the three enzymes, which reached statistical significance only for AST levels, whose mean reduction amounted to 16.8 and $10.7 \%$ for active and control groups, respectively $(P<0.04$ and $<0.02)$. Posttreatment levels did not differ between the two groups. The lack of difference was also seen in an ancillary analysis, when the three enzymes were considered as a composite endpoint, both by MANOVA and by intra-subject analyses.

The results of the secondary endpoints of the study, that is, other liver function, metabolic and inflammatory variables, did not show any difference within and between treatments, except for a slight but significant increase at T1 of HDL, LDL and total cholesterol by $7 \cdot 1,13.0$ and $8.8 \%$, respectively, and glucose by $5 \%$ in the active group.

The anthropometric measures (body weight, BMI and waist circumference) also remained unchanged in both control and active mixture supplementations, suggesting that neither group made noticeable changes in lifestyle.

Table 3 reports the circulating levels of the main markers of in vivo activation of coagulation and fibrinolysis $(\mathrm{F} 1+2$, PAP, D-dimer and TAFIa/ai), the circulating levels of factors known to influence fibrinolytic capacity (t-PA, PAI-1 and TAFI) and the results of the clot lysis assay, a global test that measures the fibrinolytic resistance of plasma clots exposed to exogenous t-PA. Thrombin generation parameters, which reflect the procoagulant capacity of plasma, are reported in Table 4. The latter parameters were also evaluated in the presence of thrombomodulin to assess the generation of thrombin in the presence of a functioning protein $\mathrm{C}$ system in order to better mimic the physiological conditions. On the whole, the baseline levels of these variables, if compared with values of healthy subjects obtained in the same laboratory using the very same experimental conditions $^{(21,22)}$, suggest an increased pro-coagulant potential in NAFLD subjects. In particular, the main thrombin generation parameters, that is, endogenous thrombin potential and peak thrombin activity, were visibly higher compared with a control group investigated in the same period using the same reagents ${ }^{(21)}$. Consistent with the majority of hepatic and inflammatory variables, the baseline coagulation-fibrinolytic data were similar in the two groups and were not affected by either treatment.

\section{Compliance to the treatment}

The distribution of missed days and returned capsules, as a measure of treatment compliance, was similar in the two treatment groups: overall $98 \%$ of the study participants were totally compliant to the therapy and assumed all the capsules; the time lapse between basal and second visit was 100 (SD 12) and $101(\mathrm{SD} 15)(P=0.46)$ in the active and control treatment groups, respectively. A subgroup analysis by excluding subjects who were available for the final visit (T1) more than $103 \mathrm{~d}$ after enrolment, therefore remaining without supplementation for at least 2 weeks, did not substantially modify the results. They were eleven and fifteen for active and control treatments, respectively.

No relevant changes in dietary habits were recorded. 
Table 2. Hepatic enzymes, metabolic and anthropometric variables at baseline and after the 3-month supplementation with active mixture or control* (Mean values and standard deviations or medians and interquartile ranges (IQR))

\begin{tabular}{|c|c|c|c|c|c|c|c|c|}
\hline \multirow[b]{2}{*}{ Variable } & & \multicolumn{2}{|c|}{ Before } & \multirow{2}{*}{$\begin{array}{c}P \text { : active } v . \\
\text { control before } \\
\text { treatment }\end{array}$} & \multicolumn{2}{|c|}{ After } & \multirow{2}{*}{$\begin{array}{c}P \text { : after } v . \\
\text { before } \\
\text { intra-subject }\end{array}$} & \multirow{2}{*}{$\begin{array}{c}P \text { : active } v . \\
\text { control (delta \%) } \\
\text { treatment }\end{array}$} \\
\hline & & $\begin{array}{l}\text { Mean or } \\
\text { median }\end{array}$ & $\begin{array}{l}\text { SD or } \\
\text { IQR }\end{array}$ & & $\begin{array}{l}\text { Mean or } \\
\text { median }\end{array}$ & $\begin{array}{l}\text { SD or } \\
\text { IQR }\end{array}$ & & \\
\hline \multirow[t]{2}{*}{ Body weight (kg) } & Control & $87 \cdot 9$ & $16 \cdot 4$ & 0.16 & $88 \cdot 0$ & $16 \cdot 7$ & 0.43 & 0.81 \\
\hline & Active & $83 \cdot 9$ & $14 \cdot 3$ & 0.10 & 83.9 & $14 \cdot 6$ & 0.57 & 0.81 \\
\hline \multirow[t]{2}{*}{ BMI $\left(\mathrm{kg} / \mathrm{m}^{2}\right)$} & Control & $31 \cdot 3$ & $5 \cdot 2$ & 0.14 & 31.4 & $5 \cdot 3$ & 0.47 & 0.47 \\
\hline & Active & $30 \cdot 1$ & 3.6 & & $30 \cdot 0$ & 3.7 & 0.94 & \\
\hline \multirow[t]{2}{*}{ Waist circumference $(\mathrm{cm})$} & Control & 106 & 13 & 0.44 & 106 & 13 & 0.46 & 0.96 \\
\hline & Active & 104 & 12 & & 104 & 12 & 0.51 & \\
\hline \multirow[t]{2}{*}{ AST (IU/I) } & Control & $37 \cdot 5$ & $25 \cdot 2$ & 0.63 & $31 \cdot 2$ & $14 \cdot 7$ & 0.02 & 0.47 \\
\hline & Active & $35 \cdot 4$ & $22 \cdot 5$ & & 31.6 & $15 \cdot 0$ & 0.04 & \\
\hline \multirow[t]{2}{*}{$\mathrm{ALT}(\mathrm{IU} / \mathrm{l})$} & Control & $48 \cdot 3$ & $43 \cdot 4$ & 0.69 & $40 \cdot 0$ & 21.9 & 0.08 & 0.07 \\
\hline & Active & $45 \cdot 5$ & $28 \cdot 4$ & & $43 \cdot 8$ & 22.9 & 0.53 & \\
\hline \multirow[t]{2}{*}{$\gamma-\mathrm{GT}(\mathrm{IU} / \mathrm{I})$} & Control & $98 \cdot 5$ & 136 & 0.90 & $75 \cdot 6$ & 64.4 & 0.21 & 0.71 \\
\hline & Active & 101.4 & 101 & & $87 \cdot 2$ & $71 \cdot 1$ & 0.07 & \\
\hline \multirow[t]{2}{*}{ Direct bilirubin $(\mu \mathrm{mol} / \mathrm{l})$} & Control & $2 \cdot 74$ & 2.91 & 0.08 & $2 \cdot 39$ & 1.71 & 0.24 & 0.04 \\
\hline & Active & 2.05 & $1 \cdot 20$ & & $2 \cdot 22$ & 1.20 & 0.09 & \\
\hline \multirow[t]{2}{*}{ Indirect bilirubin $(\mu \mathrm{mol} / \mathrm{l})$} & Control & 9.92 & 5.99 & 0.51 & $10 \cdot 09$ & $5 \cdot 30$ & 0.79 & 0.48 \\
\hline & Active & $9 \cdot 23$ & 5.47 & & $9 \cdot 23$ & $5 \cdot 64$ & 0.97 & \\
\hline \multirow[t]{2}{*}{ Total bilirubin $(\mu \mathrm{mol} / \mathrm{l})$} & Control & 12.65 & $7 \cdot 87$ & 0.27 & $12 \cdot 48$ & $6 \cdot 50$ & 0.76 & 0.90 \\
\hline & Active & $11 \cdot 29$ & 5.99 & & 11.46 & $6 \cdot 33$ & 0.70 & \\
\hline \multirow[t]{2}{*}{ HDL-cholesterol (mmol/l) } & Control & 1.25 & 0.46 & 0.62 & 1.25 & 0.33 & 0.91 & 0.43 \\
\hline & Active & $1 \cdot 21$ & 0.39 & & $1 \cdot 30$ & 0.36 & 0.009 & \\
\hline \multirow[t]{2}{*}{ LDL-cholesterol (mmol/l) } & Control & $2 \cdot 87$ & $1 \cdot 19$ & 0.67 & 3.03 & $1 \cdot 11$ & 0.14 & 0.75 \\
\hline & Active & 2.97 & 1.27 & & 3.36 & 1.27 & 0.002 & \\
\hline \multirow[t]{2}{*}{ Total cholesterol (mmol/l) } & Control & 4.86 & $1 \cdot 24$ & 0.60 & 4.99 & $1 \cdot 19$ & 0.26 & 0.11 \\
\hline & Active & 4.99 & 1.58 & & 5.43 & 1.47 & 0.004 & \\
\hline \multirow[t]{2}{*}{ TAG $(\mathrm{mmol} / \mathrm{l})$} & Control & $1 \cdot 22$ & $0.93-1.75$ & 0.21 & 1.47 & $0.93-1.90$ & 0.40 & 0.52 \\
\hline & Active & 1.52 & $1 \cdot 16-2 \cdot 12$ & & 1.51 & $1.07-2.19$ & 0.89 & \\
\hline \multirow[t]{2}{*}{ Glucose (mmol/l) } & Control & 5.44 & $4 \cdot 72-6 \cdot 27$ & 0.97 & $5 \cdot 27$ & $4.50-6.66$ & 0.46 & 0.39 \\
\hline & Active & $5 \cdot 11$ & $4.50-6.44$ & & 5.49 & $4.83-6.94$ & 0.03 & \\
\hline \multirow[t]{2}{*}{ Insulin (pmol/l) } & Control & 115 & 72.9 & 0.12 & 120 & $67 \cdot 4$ & 0.50 & 0.55 \\
\hline & Active & 145 & 118 & & 180 & 379 & 0.57 & \\
\hline \multirow[t]{2}{*}{ HOMA-IR } & Control & 3.84 & $2 \cdot 02-7 \cdot 80$ & 0.50 & 4.02 & $2 \cdot 69-6 \cdot 29$ & 0.71 & 0.33 \\
\hline & Active & 3.63 & $2 \cdot 17-5 \cdot 76$ & & 3.99 & $2 \cdot 08-6.63$ & 0.08 & \\
\hline \multirow[t]{2}{*}{ C-reactive protein $(\mathrm{nmol} / \mathrm{l})$} & Control & $28 \cdot 3$ & $3.4-44.5$ & 0.44 & $24 \cdot 0$ & $13 \cdot 3-41 \cdot 0$ & 0.43 & 0.56 \\
\hline & Active & 21.0 & $13 \cdot 3-38 \cdot 1$ & & $17 \cdot 1$ & $12 \cdot 9-30 \cdot 5$ & 0.12 & \\
\hline
\end{tabular}

AST, aspartate aminotransferase; ALT, alanine aminotransferase; $\gamma$-GT, $\gamma$-glutamyl transpeptidase; HOMA-IR, homeostatic model assessment of insulin resistance.

${ }^{*}$ Number of subjects $=58$ and 55 , for control and active treatment groups, respectively.

The reported complaints of adverse effects were dermatitis (in one subject who abandoned the study), sudden increase in body weight $(n 1)$, night pyrosis $(n 1)$, abdominal bloating $(n 1)$ in the active group, abnormal faeces colour ( $n 1$, dropout subject) and dysenteriae ( $n 1)$ in the control group.

\section{Discussion}

Our study of adult subjects with NAFLD tested a novel multicomponent mixture, selected on the basis of previous knowledge, with the potential advantage to target different mechanisms and to utilise relatively low doses of each compound. The chronic supplementation with the nutraceutical mixture was well tolerated and apparently safe in NAFLD subjects: the adverse effects reported were of relatively low severity and the compliance to treatment was high. Choline was used both in the control and in the active mixture at a dose of $43 \mathrm{mg} / \mathrm{d}$, which is relatively low compared with the minimal dose reported in the EFSA's health claim, and corresponds to $15 \%$ of the median daily intake of an adult subject. Its presence in the active mixture was expected to facilitate intracellular transport of other compounds, such as silymarin and curcumin.
Despite these promising grounds, we did not observe any significant effect of the multicomponent nutraceutical on liver enzymes. In fact, the average reductions in hepatic enzymes after treatment ranged from 23.2 to $3.7 \%$, independent of the type of supplement received but did not reach statistical significance (except for AST) nor discriminated subjects between active and control supplement groups. Most likely, the observed improvement in AST was due to choline, which was present in both intervention arms. However, the possibility of a 'spontaneous' or casual effect cannot be excluded because we did not include a true placebo group.

Other liver function and metabolic parameters were also unaffected by the supplementation. An unexpected finding of our study was a minor (less than $10 \%$ ) increase in serum cholesterol (total, LDL and HDL) and glucose levels in the subjects randomised to the active mixture. Within the different compounds present in the mixture tested in the present study, DHA has been shown to increase LDL-cholesterol particle size $\mathrm{e}^{(23)}$ and to down-regulate receptor-mediated LDL-cholesterol clearance, in part via reduced expression of LDL receptors by hepatocytes. These data, however, were not confirmed by other studies $^{(24)}$. 
Table 3. Haemostatic variables at baseline and after the 3-month supplementation with active mixture or control* (Mean values and standard deviations)

\begin{tabular}{|c|c|c|c|c|c|c|c|c|}
\hline \multirow[b]{2}{*}{ Variable } & & \multicolumn{2}{|c|}{ Before } & \multirow{2}{*}{$\begin{array}{c}P \text { : active } v \text {. control } \\
\text { before treatment }\end{array}$} & \multicolumn{2}{|c|}{ After } & \multirow{2}{*}{$\begin{array}{c}P \text { : after } v \text {. before } \\
\text { intra-subject }\end{array}$} & \multirow{2}{*}{$\begin{array}{l}P \text { : active } v \text {. control } \\
\text { (delta \%) treatment }\end{array}$} \\
\hline & & Mean & SD & & Mean & SD & & \\
\hline \multirow{2}{*}{$\mathrm{F}_{1+2}(\mathrm{pmol} / \mathrm{l})$} & Control & 258 & 187 & 0.97 & 291 & 265 & $0 \cdot 31$ & 0.92 \\
\hline & Active & 259 & 160 & & 270 & 164 & 0.69 & \\
\hline \multirow[t]{2}{*}{ PAP (ng/ml) } & Control & 365 & 176 & 0.80 & 332 & 106 & 0.06 & 0.83 \\
\hline & Active & 373 & 150 & & 359 & 134 & 0.39 & \\
\hline \multirow[t]{2}{*}{ D-Dimer (ng/ml) } & Control & 347 & 233 & 0.82 & 321 & 161 & 0.34 & 0.68 \\
\hline & Active & 357 & 211 & & 329 & 170 & 0.07 & \\
\hline \multirow{2}{*}{ t-PA (ng/ml) } & Control & $10 \cdot 1$ & $9 \cdot 0$ & 0.27 & 9.9 & 8.9 & 0.49 & 0.56 \\
\hline & Active & $8 \cdot 6$ & $4 \cdot 8$ & & 8.7 & 4.9 & 0.77 & \\
\hline \multirow[t]{2}{*}{ PAl-1 (ng/ml) } & Control & 48.0 & 18.5 & 0.15 & $47 \cdot 7$ & 21.9 & 0.91 & 0.13 \\
\hline & Active & $42 \cdot 7$ & $19 \cdot 8$ & & 44.3 & 18.4 & 0.49 & \\
\hline \multirow[t]{2}{*}{ TAFI (\%) } & Control & 100 & 23 & 0.28 & 99 & 21 & 0.52 & 0.93 \\
\hline & Active & 105 & 23 & & 104 & 23 & 0.55 & \\
\hline \multirow[t]{2}{*}{ TAFla/ai (ng/ml) } & Control & 13.8 & 5.5 & 0.42 & 14.0 & 4.8 & 0.67 & 0.48 \\
\hline & Active & 14.6 & $5 \cdot 2$ & & $19 \cdot 7$ & 41.5 & 0.35 & \\
\hline \multirow[t]{2}{*}{ Clot lysis time (min) } & Control & 158 & 111 & 0.95 & 167 & 150 & 0.24 & 0.68 \\
\hline & Active & 159 & 163 & & 136 & 82 & 0.30 & \\
\hline
\end{tabular}

PAP, plasmin- $\alpha 2$-antiplasmin complex; t-PA, tissue plasminogen activator; PAl-1, plasminogen activator inhibitor-1; TAFI, thrombin activatable fibrinolysis inhibitor; TAFla, activated TAFI; TAFla/ai, a combination of TAFla and its inactive derivative TAFlai.

${ }^{*}$ Number of subjects $=58$ and 55 , for control and active treatment groups, respectively.

Table 4. Thrombin generation parameters at baseline and after the 3-month supplementation with active mixture or control ${ }^{\star}$ (Mean values and standard deviations)

\begin{tabular}{|c|c|c|c|c|c|c|c|c|}
\hline \multirow[b]{2}{*}{ Parameter } & & \multicolumn{2}{|c|}{ Before } & \multirow{2}{*}{$\begin{array}{c}P \text { : active } v . \\
\text { control before } \\
\text { treatment }\end{array}$} & \multicolumn{2}{|c|}{ After } & \multirow{2}{*}{$\begin{array}{c}P \text { : after } v \text {. before } \\
\text { intra-subject }\end{array}$} & \multirow{2}{*}{$\begin{array}{c}P \text { : active } v \text {. } \\
\text { control (delta \% } \\
\text { treatment }\end{array}$} \\
\hline & & Mean & SD & & Mean & SD & & \\
\hline \multicolumn{9}{|c|}{ Without thrombomodulin } \\
\hline Lag time $(\min )$ & $\begin{array}{l}\text { Control } \\
\text { Active }\end{array}$ & $\begin{array}{l}2.60 \\
2.67\end{array}$ & $\begin{array}{l}1 \cdot 16 \\
1.17\end{array}$ & 0.74 & $\begin{array}{l}2.51 \\
2.41\end{array}$ & $\begin{array}{l}1.11 \\
0.82\end{array}$ & $\begin{array}{l}0.13 \\
0.02\end{array}$ & 0.50 \\
\hline Peak (nм) & $\begin{array}{l}\text { Control } \\
\text { Active }\end{array}$ & $\begin{array}{l}243 \\
248\end{array}$ & $\begin{array}{l}62 \\
78\end{array}$ & 0.72 & $\begin{array}{l}239 \\
238\end{array}$ & $\begin{array}{l}63 \\
74\end{array}$ & $\begin{array}{l}0.43 \\
0.11\end{array}$ & 0.24 \\
\hline TTPeak (min) & $\begin{array}{l}\text { Control } \\
\text { Active }\end{array}$ & $\begin{array}{l}6 \cdot 0 \\
6 \cdot 1\end{array}$ & $\begin{array}{l}1.9 \\
2.4\end{array}$ & 0.77 & $\begin{array}{l}5 \cdot 8 \\
5.8\end{array}$ & $\begin{array}{l}1.9 \\
1.6\end{array}$ & $\begin{array}{l}0.18 \\
0.31\end{array}$ & 0.36 \\
\hline Velocity $(\mathrm{nm} / \mathrm{min})$ & $\begin{array}{l}\text { Control } \\
\text { Active }\end{array}$ & $\begin{array}{l}81 \cdot 0 \\
84.4\end{array}$ & $\begin{array}{l}35.6 \\
44.8\end{array}$ & 0.66 & $\begin{array}{l}82 \cdot 7 \\
80 \cdot 2\end{array}$ & $\begin{array}{l}38.8 \\
41.8\end{array}$ & $\begin{array}{l}0.54 \\
0.15\end{array}$ & 0.23 \\
\hline ETP (nм x min) & $\begin{array}{l}\text { Control } \\
\text { Active }\end{array}$ & $\begin{array}{l}1412 \\
1403\end{array}$ & $\begin{array}{l}259 \\
305\end{array}$ & 0.87 & $\begin{array}{l}1386 \\
1382\end{array}$ & $\begin{array}{l}244 \\
287\end{array}$ & $\begin{array}{l}0.19 \\
0.45\end{array}$ & 0.24 \\
\hline \multicolumn{9}{|l|}{ With thrombomodulin } \\
\hline Lag time (min) & $\begin{array}{l}\text { Control } \\
\text { Active }\end{array}$ & $\begin{array}{l}2.73 \\
2.74\end{array}$ & $\begin{array}{l}1.37 \\
1.14\end{array}$ & 0.95 & $\begin{array}{l}2.58 \\
2.52\end{array}$ & $\begin{array}{l}1.2 \\
0.9\end{array}$ & $\begin{array}{l}0.10 \\
0.07\end{array}$ & 0.41 \\
\hline Peak (nм) & $\begin{array}{l}\text { Control } \\
\text { Active }\end{array}$ & $\begin{array}{l}176 \\
181\end{array}$ & $\begin{array}{l}73 \\
90\end{array}$ & 0.77 & $\begin{array}{l}174 \\
173\end{array}$ & $\begin{array}{l}76 \\
87\end{array}$ & $\begin{array}{l}0.69 \\
0.25\end{array}$ & 0.41 \\
\hline TTPeak (min) & $\begin{array}{l}\text { Control } \\
\text { Active }\end{array}$ & $\begin{array}{l}5 \cdot 7 \\
5 \cdot 7\end{array}$ & $\begin{array}{l}1.8 \\
1.7\end{array}$ & 0.92 & $\begin{array}{l}5.5 \\
5.5\end{array}$ & $\begin{array}{l}1.9 \\
1.3\end{array}$ & $\begin{array}{l}0.12 \\
0.18\end{array}$ & 0.17 \\
\hline Velocity (nм/min) & $\begin{array}{l}\text { Control } \\
\text { Active }\end{array}$ & $\begin{array}{l}66 \cdot 2 \\
69 \cdot 4\end{array}$ & $\begin{array}{l}37.8 \\
44.0\end{array}$ & 0.68 & $\begin{array}{l}67 \cdot 6 \\
67 \cdot 4\end{array}$ & $\begin{array}{l}39.6 \\
44.6\end{array}$ & $\begin{array}{l}0.62 \\
0.39\end{array}$ & 0.17 \\
\hline ETP (nм x min) & $\begin{array}{l}\text { Control } \\
\text { Active }\end{array}$ & $\begin{array}{l}898 \\
877\end{array}$ & $\begin{array}{l}334 \\
364\end{array}$ & 0.75 & $\begin{array}{l}854 \\
844\end{array}$ & $\begin{array}{l}316 \\
371\end{array}$ & $\begin{array}{l}0.19 \\
0.26\end{array}$ & $0 \cdot 16$ \\
\hline
\end{tabular}

TTPeak, time to peak; ETP, endogenous thrombin potential.

${ }^{*}$ Number of subjects $=58$ and 55 for control and active treatment groups, respectively.

Liver is the source and the site of clearance of most clotting/ fibrinolytic factors (both activators and inhibitors), and a progressive pro-coagulant imbalance has been reported in liver disorders, suggesting a pathogenetic role of the prothrombotic shift in NAFLD-associated risk of CVD and fibrosis progres$\operatorname{sion}^{(10-12)}$. For this reason, we performed an in-depth study of coagulation and fibrinolysis, through the use of global tests (i.e. thrombin generation and clot lysis), which gives an overall picture of the prothrombotic potential of plasma, and through the assay of specific factors and activation markers, which reflect the in vivo activation of coagulation and fibrinolysis. On the whole, the data obtained point to a heightened prothrombotic potential in NAFLD. Even though our trial did not include a control group of healthy subjects, the marked difference in the results obtained in normal subjects by the same laboratory, using the same methods and reagents ${ }^{(21,22)}$, provides an indirect support to this conclusion. Besides this aspect, which needs to be confirmed by a head-to-head comparison, our study shows that 
none of the variables tested was affected by supplementation with active or control nutraceuticals. It should be stressed that in a recent study ${ }^{(21)}$ we could demonstrate a significant reduction in the prothrombotic potential (e.g. reduced thrombin generation, faster clot lysis) of healthy subjects following a 4-week intake of fresh grape, showing that some of the variables assayed in the present study can be influenced by dietary supplementation. The latter finding, along with the array of variables measured in the present study, allows us to conclude that the nutraceutical formulation tested in this trial has no antithrombotic effect, at least after a 3-month period of treatment.

NAFLD is a condition characterised by a wide spectrum of hepatic changes, which may progress into overt liver disease such as liver fibrosis and cirrhosis but is also associated with comorbidities such as insulin resistance, the metabolic syndrome, thyroid dysfunction, chronic inflammation, hypercoagulable state, chronic kidney disease, hyperuricemia and others, all conditions at increased risk of CVD and mortality. Therefore, although the mechanisms underlying the onset and progression of NAFLD are not clearly defined, several types of nutraceuticals have been proposed and for some of them clinical trials have shown an improvement of liver function tests and possibly of liver histological changes.

Nutraceutical supplementation has been previously tested in NAFLD to prevent or reduce hepatic enzyme imbalance by targeting different potential mechanisms such as insulin resistance, inflammation and oxidative stress. The most studied compounds are vitamins D, E and C, carnitine, $n$-3-fatty acids such as DHA and EPA, plant-derived polyphenols including the flavonoids silymarin and anthocyanins, curcumin and the stilbene resveratrol. Mediterranean diet has been also proposed as a potentially useful approach for NAFLD patients. The available clinical evidence on the effects and possible mechanisms of action of nutraceuticals has been recently reviewed ${ }^{(6)}$.

The following considerations may be useful for future studies. The study design, a randomised double-blind, controlled trial, was correctly applied and randomisation of patients from different centres was successful. The study was powered to detect a $20 \%$ reduction in the primary endpoints by the active supplement. Thus, the small, though significant, decrease in AST levels following both treatments may be considered casual and of little clinical relevance.

The dose of each component was selected on the basis of previous clinical studies on single ingredient or on the combination of two components. We purposely chose to reduce the concentration of each component in view of a possible multitarget potentiation, as discussed above. Concerning the duration of the study, the 3-month period was considered appropriate based on our previous experience with dietary supplementation in healthy subjects ${ }^{(21)}$ and on previously published studies ${ }^{(25,26)}$. On the other hand, a relatively short treatment period was considered suitable to guarantee a good compliance and provide a relatively rapid answer on treatment efficacy. Unfortunately, no measurements of the supplemented compounds or of their biomarkers in body fluids could be performed.

The subjects included in the trial showed an ample inter-individual variability, not only for the hepatic and metabolic markers but also for other clinical conditions and biological variables such as obesity, diabetes, inflammation markers, status of fibrosis, which could differently modulate the response to supplementation. This heterogeneity, while representing a limitation for it may decrease the statistical power of the study, was considered to better reflect real-life situation and thus may represent an advantage as to the applicability of the results to a general population.

\section{What have we learnt from the present study?}

First of all, trials may obviously end with either positive or negative results: the latter are as important as the former, as they may clarify some uncertain points on the clinical condition selected and/or its potential treatment.

Second, it may be quite difficult to show any significant decrease in biochemical parameters that are only marginally altered before the treatment. In our previous experience on other nutraceuticals, we had observed a measurable effect of these compounds only in 'stressed' conditions, such as challenging healthy volunteers with a fatty meal, that induces an acute increase in inflammatory reactions ${ }^{(27)}$. We cannot exclude that the inclusion in the present study of patients with a more severe form of NAFLD could have produced more apparent results.

Third, in a chronic condition such as NAFLD, a longer treatment might have been preferable but could not be performed in our study for organisation reasons.

Fourth, the significant increase in cholesterol and glucose levels should caution against consuming nutraceuticals for which no evidence-based efficacy has been previously shown, because some unexpected, though minor, side effects might occur.

In conclusion, in the present trial we were unable to show any effect of a mixture of dietary natural compounds on different relevant physiopathological markers of NAFLD. The results of our study, as well as our protocol choices, may be useful for future trials, which will have to take into account the number of subjects, the inclusion criteria (to obtain a more homogeneous sample, preferably at higher risk), the nature and the concentration of nutraceuticals, a longer duration of treatment, along with other aspects such as the gut microbiota interference in lipid metabolism.

\section{Acknowledgements}

We thank doctors and nurses of the recruiting sites for the valuable support provided to complete the present study.

The present study was supported by the Italian Ministry of University and Research (MIUR, PON01_01226/1 - Decr. N.l/ Ric 18-1-2010).

G. d. G., L. I. and N. S. designed the study and revised the manuscript; C. C. prepared the protocol and documents for ethical committee approval and trial registration, supervised all the aspects of the study and wrote the manuscript; M. C. supervised the coagulation-fibrinolysis concepts, tests and writing and supervised trial completion at the Bari sites; M. S., F. S., C. T. A. and F. I. performed the laboratory tests; S. C. and A. D. C. performed the statistical analyses. G. D. B., P. P. and M. B. were responsible for recruitment of subjects for the trial. All authors approved the final version of the manuscript. 
All authors declare that they have no conflicts of interest. The Company who provided the capsules tested in the trial had no role in the study design, collection, analysis and interpretation of data, nor in the decision to submit a manuscript for publication and in its writing.

\section{Ethical statement}

The study protocol was approved by the ethical committee of the IRCCS Neuromed, the trial Coordinator's Institution, followed by each recruitment centre's Ethical Committee, namely, the Azienda Sanitaria Regionale del Molise (ASReM) and the Azienda Ospedaliero-Universitaria Policlinico of Bari.

\section{Informed consent}

Prior to the study, all participating subjects signed a written

informed consent approved by the ethical committees.

\section{References}

1. Araújo AR, Rosso N, Bedogni G, et al. (2018) Global epidemiology of non-alcoholic fatty liver disease/non-alcoholic steatohepatitis: what we need in the future. Liver Int 38, Suppl. 1, 47-51.

2. Bellentani S (2017) The epidemiology of non-alcoholic fatty liver disease. Liver Int 37, Suppl. 1, 81-84.

3. Younossi ZM, Koenig AB, Abdelatif D, et al. (2016) Global epidemiology of nonalcoholic fatty liver disease-meta-analytic assessment of prevalence, incidence, and outcomes. Hepatology 64, 73-84.

4. Stefan N, Häring HU \& Cusi K (2019) Non-alcoholic fatty liver disease: causes, diagnosis, cardiometabolic consequences, and treatment strategies. Lancet Diabetes Endocrinol 7, 313-324.

5. Chalasani N, Younossi Z, Lavine JE, et al. American Association for the Study of Liver Diseases; American College of Gastroenterology; American Gastroenterological Association (2012) The diagnosis and management of non-alcoholic fatty liver disease: practice guideline by the American Association for the Study of Liver Diseases, American College of Gastroenterology, and the American Gastroenterological Association. Am J Gastroenterol 107, 811-826.

6. Cicero AFG, Colletti A \& Bellentani S (2018) Nutraceutical Approach to Non-Alcoholic Fatty Liver Disease (NAFLD): the available clinical evidence. Nutrients 10, E1153.

7. Loguercio C, Andreone P, Brisc C, et al. (2012) Silybin combined with phosphatidylcholine and vitamin $\mathrm{E}$ in patients with nonalcoholic fatty liver disease: a randomized controlled trial. Free Radic Biol Med 52, 1658-1665.

8. EFSA Panel on Dietetic Products, Nutrition and Allergies (NDA) (2011) Scientific opinion on the substantiation of health claims related to choline and contribution to normal lipid metabolism (ID 3186) and maintenance of normal liver function (ID 1501). EFSA J 9, 2056.

9. Stellavato A, Pirozzi AVA, de Novellis F, et al. (2018) In vitro assessment of nutraceutical compounds and novel nutraceutical formulations in a liver-steatosis-based model. Lipids Health Dis 17, 24.
10. Tripodi A, Fracanzani AL, Primignani M, et al. (2014) Procoagulant imbalance in patients with non-alcoholic fatty liver disease. J Hepatol 61, 148-154.

11. Northup PG, Argo CK, Shah N, et al. (2012) Hypercoagulation and thrombophilia in non-alcoholic fatty liver disease: mechanisms, human evidence, therapeutic implications, and preventive implications. Semin Liver Dis 32, 39-48.

12. Potze W, Siddiqui MS, Boyett SL, et al. (2016) Preserved hemostatic status in patients with non-alcoholic fatty liver disease. J Hepatol 65, 980-987.

13. Schulz KF, Altman DG, Moher D, et al. (2010) CONSORT 2010 statement: updated guidelines for reporting parallel group randomised trials. BMJ 340, c332.

14. Saverymuttu SH, Joseph AE \& Maxwell JD (1986) Ultrasound scanning in the detection of hepatic fibrosis and steatosis. BMJ 292, 13-15.

15. Leoni S, Tovoli F, Napoli L, et al. (2018) Current guidelines for the management of non-alcoholic fatty liver disease: a systematic review with comparative analysis. World J Gastroenterol 24, 3361-3373.

16. Abenavoli L, Di Renzo L, Boccuto L, et al. (2018) Health benefits of Mediterranean diet in nonalcoholic fatty liver disease. Expert Rev Gastroenterol Hepatol 12, 873-881.

17. Angulo P, Hui JM, Marchesini G, et al. (2007) The NAFLD fibrosis score: a noninvasive system that identifies liver fibrosis in patients with NAFLD. Hepatology 45, 846-854.

18. Wallace TM, Levy JC \& Matthews DR (2004) Use and abuse of HOMA modeling. Diabetes Care 27, 1487-1495.

19. Semeraro F, Incampo F, Ammollo CT, et al. (2016) Dabigatran but not rivaroxaban or apixaban treatment decreases fibrinolytic resistance in patients with atrial fibrillation. Thromb Res 138, 22-29.

20. Semeraro F, Colucci M, Caironi P, et al. (2018) Platelet drop and fibrinolytic shutdown in patients with sepsis. Crit Care Med $\mathbf{4 6}$, e221-e228

21. Ammollo CT, Semeraro F, Milella RA, et al. (2017) Grape intake reduces thrombin generation and enhances plasma fibrinolysis. Potential role of circulating procoagulant microparticles. J Nutr Biochem 50, 66-73.

22. Incampo F, Carrieri C, Galasso R, et al. (2013) Effect of warfarin treatment on thrombin activatable fibrinolysis inhibitor (TAFI) activation and TAFI-mediated inhibition of fibrinolysis. $J$ Thromb Haemost 11, 315-324.

23. Theobald HE, Chowienczyk PJ, Whittall R, et al. (2004) LDL cholesterol-raising effect of low-dose docosahexaenoic acid in middle-aged men and women. Am J Clin Nutr 79, 558-563.

24. Jacobson TA, Glickstein SB, Rowe JD, et al. (2012) Effects of eicosapentaenoic acid and docosahexaenoic acid on low-density lipoprotein cholesterol and other lipids: a review. J Clin Lipidol 6, 5-18.

25. Gajos G, Zalewski J, Rostoff P, et al. (2011) Reduced thrombin formation and altered fibrin clot properties induced by polyunsaturated omega-3 fatty acids on top of dual antiplatelet therapy in patients undergoing percutaneous coronary intervention (OMEGA-PCI clot). Arterioscler Thromb Vasc Biol 31, 1696-1702.

26. Sanchez C, Poggi M, Morange PE, et al. (2012) Diet modulates endogenous thrombin generation, a biological estimate of thrombosis risk, independently of the metabolic status. Arterioscler Thromb Vasc Biol 32, 2394-2404.

27. Cerletti C, Gianfagna F, Tamburrelli C, et al. (2015) Orange juice intake during a fatty meal consumption reduces the postprandial low-grade inflammatory response in healthy subjects. Thromb Res 135, 255-259. 